\title{
Effect of Cold Rolling on Microstructure and Mechanical Properties of a Fe-23Mn-0.3C-1.5Al TWIP Steel
}

\author{
Pavel Kusakin ${ }^{1, \mathrm{a}}$, Andrey Belyakov ${ }^{1, \mathrm{~b}}$, Rustam Kaibyshev ${ }^{1, \mathrm{c}}$, Dmitri Molodov ${ }^{2, \mathrm{~d}}$ \\ ${ }^{1}$ Belgorod State University, Pobeda 85, Belgorod, 308015, Russia \\ ${ }^{2}$ Institute of Physical Metallurgy and Metal Physics, RWTH Aachen University, 52056 Aachen, \\ Germany \\ akusakin@bsu.edu.ru, bbelyakov@bsu.edu.ru, ${ }^{\mathrm{c}}$ rustam_kaibyshev@bsu.edu.ru, \\ cmolodov@imm.rwth-aachen.de
}

Keywords: TWIP-steel, deformation twinning, microstructure, mechanical properties

\begin{abstract}
Effect of cold rolling on the microstructure and mechanical properties of a Fe-23Mn0.3C-1.5Al (in wt. \%) TWIP steel with an initial grain size of $24 \mu \mathrm{m}$ was studied. Extensive deformation twinning occurred upon reduction by rolling. The volume fraction of the deformation twins attained about 0.2 at a reduction of $20 \%$. Then, the intensity of deformation twinning gradually decreased with increasing the total rolling reduction. The average twin thickness of about $20 \mathrm{~nm}$ remained unchanged, although the distance between twins progressively reduced with increasing strain. The deformation banding was observed after a reduction of $60 \%$. The thickness and volume fraction of microshear bands increased with increasing rolling reduction. The cold rolling led to significant strengthening of the steel that is accompanied by a drop on ductility. The yield stress (YS) increased from $235 \mathrm{MPa}$ in the initial state to $1400 \mathrm{MPa}$ after cold rolling with a reduction of $80 \%$, whereas the elongation to failure decreased from $96 \%$ to $4 \%$, respectively.
\end{abstract}

\section{Introduction}

An increase in the structural strength of automotive parts is an attractive way to reduce vehicle weight, that provides lower fuel consumption and exhaust emissions [1,2]. In addition, the use of high-strength steels enhances the safety of passengers. High-Mn steels exhibiting the effect of twinning induced plasticity (TWIP) are very promising material for vehicle body components due to their unique combination of strength and ductility resulting from the excellent deformation hardening [3,4]. The TWIP-effect is attributed to the formation of deformation twins with nanometer thickness. Extensive twinning is observed in steels with medium stacking fault energy ranging from 20 to $40 \mathrm{~mJ} / \mathrm{m}^{2}$ [5], which in turn depends on the chemical composition. Austenitic steels exhibit high Hall-Petch response and extensive formation of nanotwins leads to the so-called dynamic Hall-Petch effect resulting in an exceptionally high strain hardening [6].

The practical applications of TWIP steels require detailed analysis of the mechanisms of microstructure evolution during extensive rolling and careful analysis of the relationships between deformation structures and mechanical properties. Recent studies of TWIP steels with different Mn content showed the following structural evolution during cold rolling takes place $[1,7,8]$. A rapid increase in the dislocation density takes place initially. Then the deformation twinning progressively develops throughout the deformation microstructures at low to medium strains. Finally, the shear banding occurs at rather large strains. The development of shear bands at large strains leads to rearrangement of twin lamellae along the rolling plane. The aim of the present work was to examine the microstructure evolution during cold rolling and its effect on mechanical properties in advanced TWIP steel. 


\section{Experimental}

An ingot of TWIP steel with a chemical composition given in Table 1 was subjected to solution treatment at $1150^{\circ} \mathrm{C}$ for 4 hours followed by forging from $140 \mathrm{~mm}$ to $50 \mathrm{~mm}$ thickness in 3 passes and subsequent final annealing at $1150^{\circ} \mathrm{C}$ for 4 hours. The forged steel was hot rolled at an initial temperature of $1150^{\circ} \mathrm{C}$ to $10 \mathrm{~mm}$ thickness and then annealed at the same temperature during 1 hour. This thermo-mechanical processing resulted in the formation of uniform microstructure composed of equiaxed grains with an average size of $24 \mu \mathrm{m}$. This material was used as the starting material. The plate samples were cold rolled to reductions 20,40,60 and $80 \%$. The rolling direction was the same as that in the hot rolling. For structural characterization the thin foils of $3 \mathrm{~mm}$ diameter were cut out parallel to RD-ND plane and grinded to $0.1 \mathrm{~mm}$ thickness. Then the discs were polished using a double jet TENUPOL-5 electrolytic polisher at voltage of $20 \mathrm{~V}$ at room temperature using an electrolyte containing 10\% perchloric acid and $90 \%$ acetic acid. The foils were examined using a JEOL JEM-2100 transmission electron microscope (TEM) operated at an acceleration voltage of $200 \mathrm{kV}$. The dislocation density was determined by analysis of X-Ray diffraction profiles using an ARL-Xtra diffractometer and $\mathrm{Cu} K \alpha$ radiation. The value of the dislocation density $\rho$ was calculated from the average values of the crystallite size $D$ and microstrain $<\varepsilon^{2}>$ by using the following relationship [9]:

$\rho=\frac{3 \sqrt{2 \pi}<\varepsilon_{50}^{2}>}{\mathrm{Db}}$

where $b$ is the Burgers vector $(b=a / \sqrt{ } 2$ for the FCC structure where $a$ is the lattice parameter). Tensile tests were carried out by using an Instron 5882 testing machine on specimens with a gauge section of $1.5 \mathrm{~mm} \times 3 \mathrm{~mm}$ cut out parallel to the rolling plane.

Table 1. Chemical composition [wt.\%] of the investigated steel

\begin{tabular}{|c|c|c|c|c|c|c|c|}
\hline $\mathrm{C}$ & $\mathrm{Mn}$ & $\mathrm{Al}$ & $\mathrm{Si}$ & $\mathrm{Cr}$ & $\mathrm{S}$ & $\mathrm{P}$ & $\mathrm{Fe}$ \\
\hline 0.304 & 23.1 & 1.5 & 0.09 & 0.08 & 0.006 & 0.017 & bal. \\
\hline
\end{tabular}

\section{Microstructure Evolution}

The cold rolling provides a rapid increase of the dislocation density and extensive deformation twinning in some favorably oriented grains. Figure 1a shows an example of bright-field image (BF) of deformation structure after a rolling reduction of $20 \%$. The grain on the left side in Figure 1a is a twinned grain as it was proved by selected area diffraction pattern indicating the $\left[\begin{array}{lll}1 & 1 & 0\end{array}\right]$ zone axes (Fig. 1b), while the grain on the right side does not contain deformation twins. The dark-field image in Fig. 1c suggests that the deformation twins appear as bundles of thin and straight twins. An average thickness of the deformation twins is about $20 \mathrm{~nm}$ and an average distance between twins is $\sim 200 \mathrm{~nm}$ at a strain of $20 \%$. Further rolling leads to flattening and elongation of initial grain towards the rolling direction. At a reduction of $40 \%$, the deformation twins appear in almost all grains. Multiple deformation twining results in the development of complicated microstructures composed of frequently intersected twins belonging to different twinning systems (Fig. 2). The deformation twins tend to rearrange along the rolling plane.

Further increasing strain leads to the appearance of microshear bands, which pass over a grain and shear the previously formed deformation twins (Fig. 3a). The thickness of shear bands tends to increase with increasing strain that leads to the development of narrow regions of localized shear.

At a reduction of $80 \%$, the mutual intersection of microshear bands with twins (Fig. 3b-e) leads to the formation of highly misoriented crystallites with a size of approx. $20 \mathrm{~nm}$ and the development of a spatial net of shear bands. Nanoscale crystallites bounded by twins rotate providing alignment of twin boundaries along the rolling plane. Figure $3 \mathrm{~d}$ indicates the [ [ $\left.\begin{array}{lll}2 & 3 & 3\end{array}\right]$ zone axis of austenite matrix with additional reflexes of [ [ $\left.\begin{array}{lll}2 & 5 & 1\end{array}\right]$ zone axis of deformation twins. The missorientation 
between the austenite matrix and twin lamellae was calculated to be $58.7^{\circ}$. Therefore, the deformation twins retain their orientation relationship within Brandon criterion even after large strain. This suggests that reorientation of twins along the rolling plane takes place like a rigid body rotation of twinned islands assisted by shear banding. In addition, twin boundaries are not transparent for gliding dislocation and the twin spacing or distance between twins can be considered as the grain size, $d$, in the Hall-Petch law.

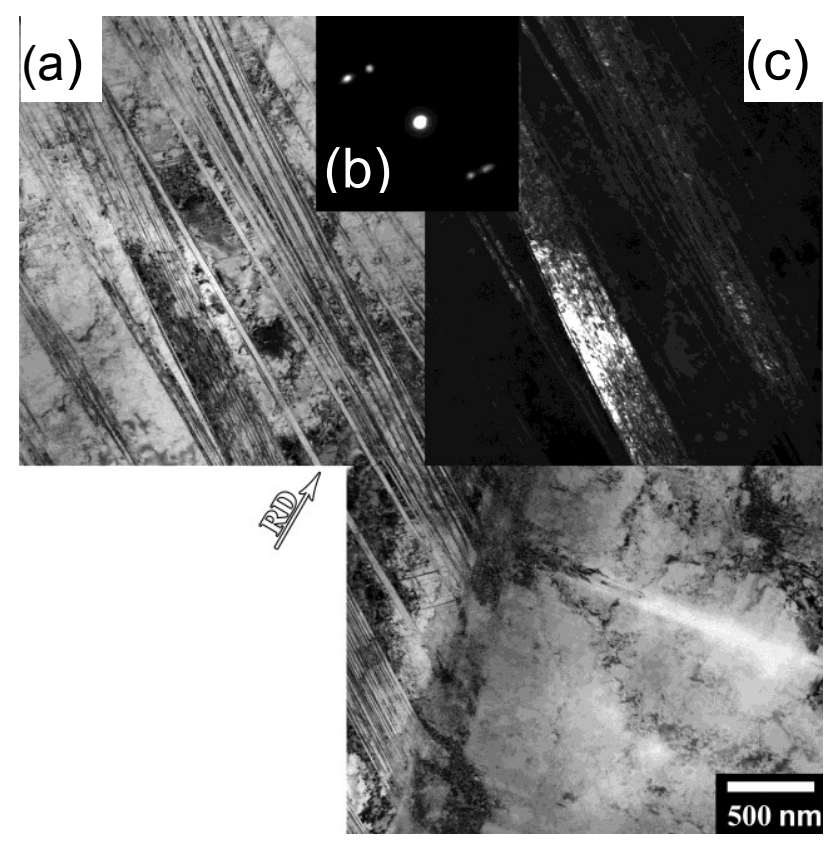

Fig. 1. TEM image of microstructure developed after $20 \%$ of cold rolling: a) BF-image, b) Diffraction pattern, c) DF-image. RD indicates the rolling direction.
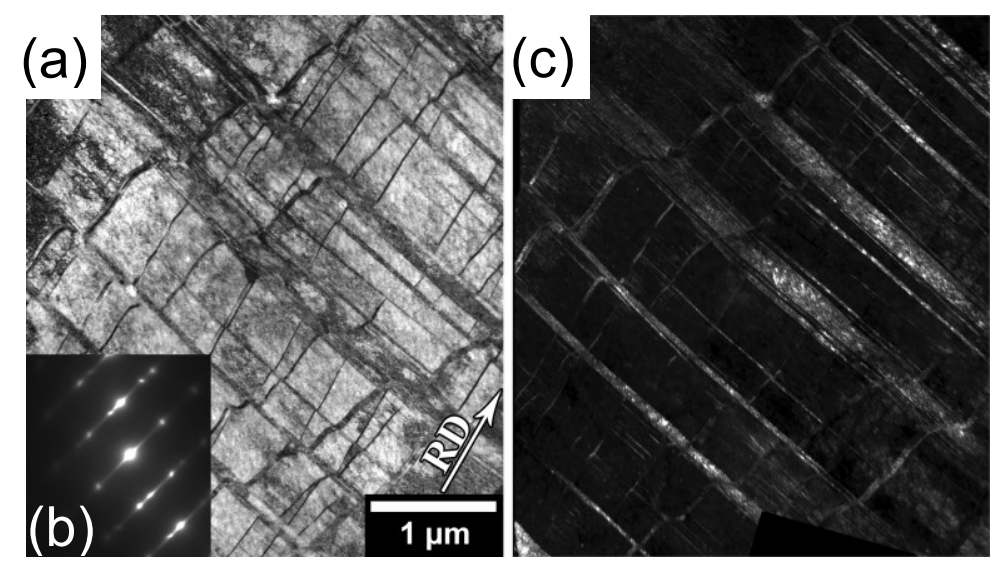

Fig. 2. TEM image of microstructure developed after $40 \%$ of cold rolling: a) BF-image, b) Diffraction pattern, c) DF-image.

Figure 4 summarizes the effect of cold rolling on some structural parameters. At a reduction of $20 \%$, the dislocation density rapidly increases to about $2 \times 10^{15} \mathrm{~m}^{-2}$ followed by slow increase during further straining and finally approaches about $4 \times 10^{15} \mathrm{~m}^{-2}$ after $80 \%$ rolling reduction. The twin thickness of $20 \mathrm{~nm}$ is almost invariant of rolling reduction. On the other hand, the distance between twins decreases during the rolling. At a reduction of 40\%, the distance between twins becomes 180 $\mathrm{nm}$. With increasing reductions from 60 to $80 \%$, the distance between deformation twins sharply decreases from 100 to $40 \mathrm{~nm}$ (Fig. 4). Therefore, the deformation twinning continuously operates during cold rolling to large strains. 

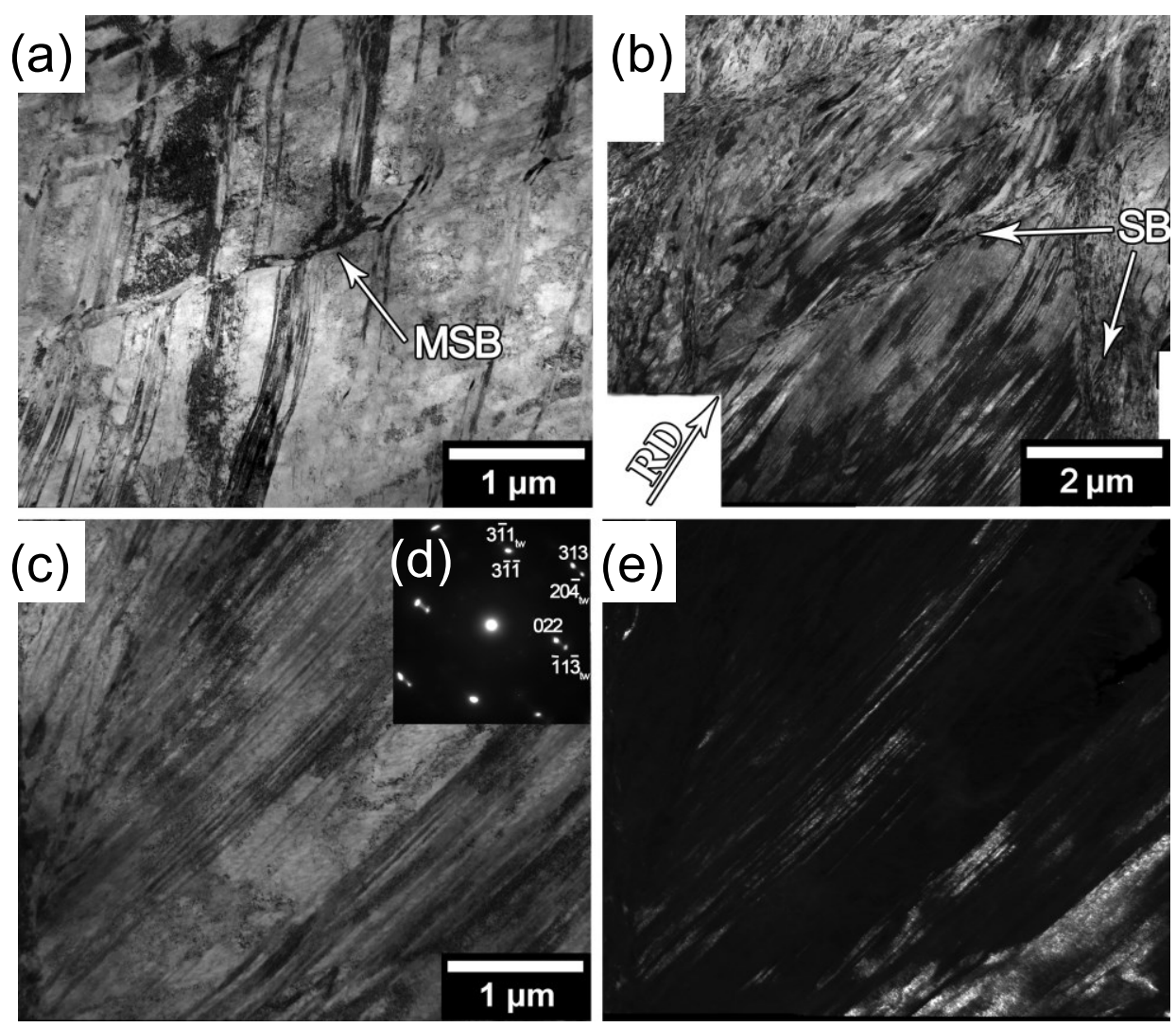

Fig. 3. TEM image of microstructure: a) BF-image of $60 \%$ cold rolled steel, b) BF-image of $80 \%$ cold rolled steel, c) BF-image of deformation twins aligned with rolling direction $(80 \%$ deformation), d) Diffraction pattern of (c), e) DF-image of (c).

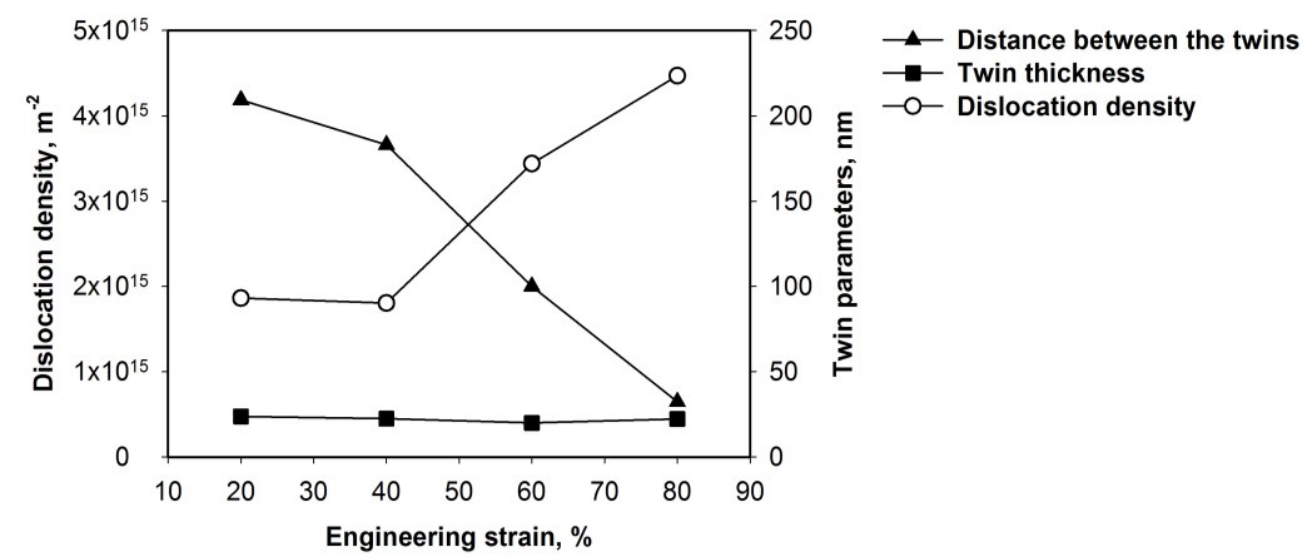

Fig. 4. Effect of cold rolling on the microstructure parameters of the Fe-23Mn-0.3C-1.5Al steel.

Figure 5 illustrates a schematic describing the evolution of the microstructure. Three welldefined stages of microstructural evolution could be distinguished. At stage I, a rapid work hardening is associated with drastic increase in the dislocation density and single twinning within separate grains. At Stage II, in the rolling reduction range of $20-40 \%$ the extensive multiple twinning occurs and density of lattice dislocations remains unchanged. The interiors of initial grains are subdivided to nanoscale crystallites having rectangular shape and delimitated by twin boundaries. At stage III, upon further rolling the evolution of microshear bands occurs concurrently with multiple twinning producing a hierarchy of grain boundary assembles. Micronscale crystallites delimited by boundaries of shear bands are subdivided to nanoscale crystallites bounded by twin boundaries. 


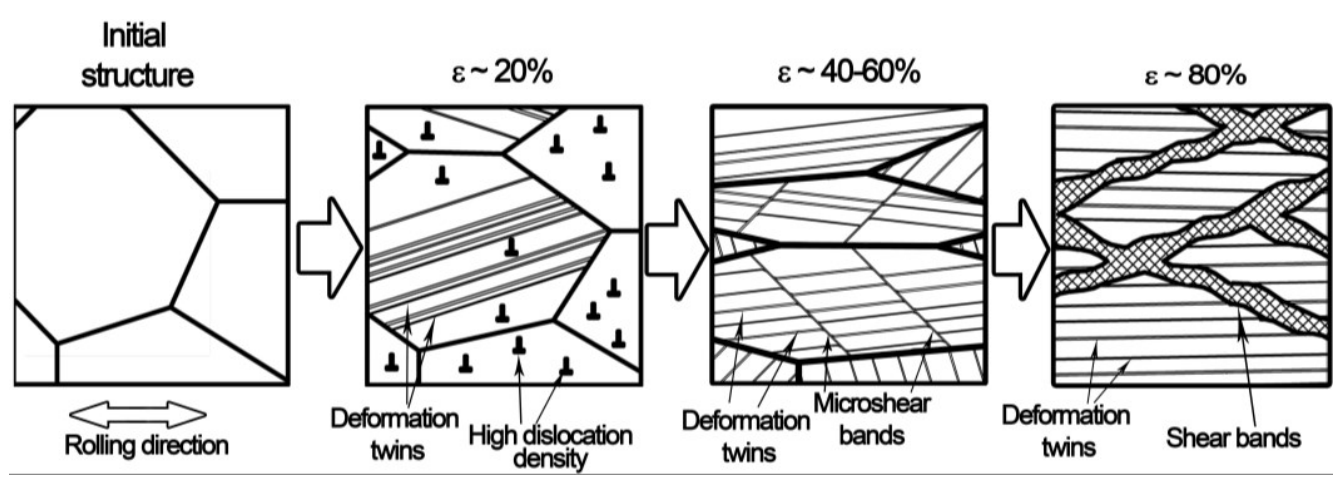

Fig. 5. Scheme of microstructure evolution during cold rolling of the Fe-23Mn-0.3C-1.5Al steel.

\section{Mechanical Properties}

In the initial annealed condition the Fe-23Mn-0.3C-1.5Al steel is characterized by a relatively low yield stress (YS) of $235 \mathrm{MPa}$ (Fig. 6). Extensive strain hardening takes place up to failure providing exceptionally high necking resistance and, therefore, very high ductility. Cold rolling highly increases YS. Ultimate tensile strength (UTS) also increases significantly, and ductility tends to drop with increasing rolling reduction (Fig. 6). After rolling reduction of $20 \%$, the sample exhibit apparent steady state flow. Samples subjected to extensive rolling with higher reductions show well-defined peak stress. After reaching a maximum stress, the flow stress progressively decreases until fracture. Increasing strain leads to progressive strain softening that highly decreases ductility owing to facilitating plastic instability. The YS increases threefold by a $20 \%$ rolling reduction. It is obvious that dislocation strengthening gives the main contribution to this increment of YS. An increase in the rolling reduction to $80 \%$ increases the YS to $1400 \mathrm{MPa}$ thus providing $+218 \%$ increase in comparison with $20 \%$ reduced sample which is attributed to the grain size strengthening in accordance with the Hall-Petch relationships, mainly. Cold rolling with a reduction of $20 \%$ leads to insignificant increase in the UTS. Further straining to $80 \%$ provides twofold increase in the UTS value. The strengthening by cold working is accompanied by remarkable degradation of the uniform elongation from $\sim 90 \%$ in the annealed condition to about $30 \%$ at a rolling reduction to $20 \%$ and then drops down to a few percent upon subsequent rolling to strains above $40 \%$.

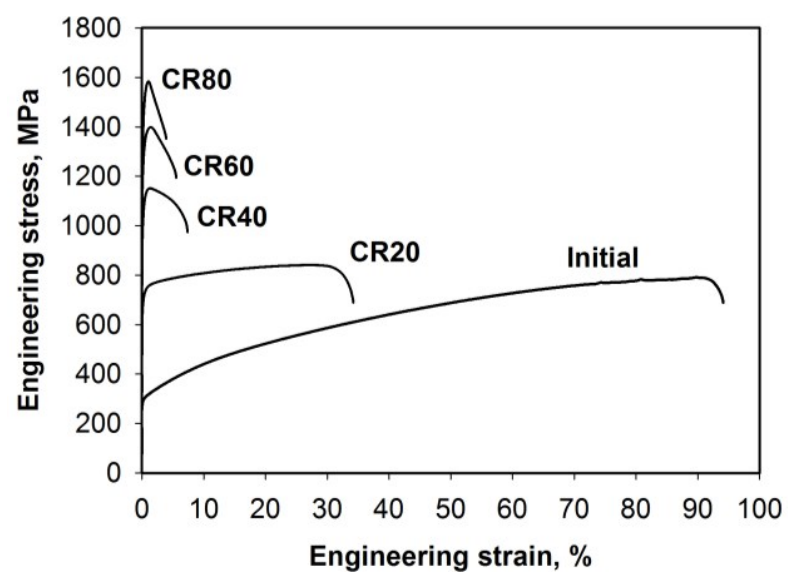

Fig. 6. The stress-strain curves of the Fe-23Mn-0.3C-1.5Al steel. Specimens marked as CR (cold rolled) with rolling reduction in $\%(40)$.

It is apparent that increments in dislocation density and density of twins under tension of the annealed steel and cold rolling with a reduction of $20 \%$ are nearly the same. This is why the UTS value of initial material and the steel rolled to a reduction of $20 \%$ are similar. Subdivision of all original grains to nanoscale crystallites after a rolling reduction of $40 \%$ and accumulation of very 
high dislocation density of well above $10^{15} \mathrm{~m}^{-2}$ after a rolling reduction of $20 \%$ makes further strain hardening during tensile tests difficult. The TWIP steel becomes susceptible to extensive necking and failure occurs in way which is much similar to various nanocrystalline materials $[10,11]$.

\section{Summary}

The deformation microstructures and mechanical properties of the $\mathrm{Fe}-23 \% \mathrm{Mn}-0.3 \% \mathrm{C}-1.5 \% \mathrm{Al}$ TWIP steel subjected to cold rolling were studied. Cold rolling with a reduction of $20 \%$ brings about high dislocation density and the formation of numerous deformation twins belonging to one system within initial grains. $+200 \%$ increase in the YS takes place, while the UTS value remains almost unchanged, and ductility decreases by a factor of $\sim 3$. Upon further rolling the multiple twinning occurs and the distance between the twins gradually decreases during cold rolling, approaching $40 \mathrm{~nm}$ after rolling reduction of $80 \%$, while the twin thickness of $20 \mathrm{~nm}$ is independent on strain. Cold rolling with reductions higher $40 \%$ leads to the development of microshear bands, which also contribute to strengthening. The deformation microstructure evolved after a rolling reduction of $80 \%$ consists of separate micron scale crystallites delimited by shear bands which are subdivided to nanoscale crystallites bounded by twin boundaries. The YS increased from $650 \mathrm{MPa}$ to $1400 \mathrm{MPa}$ with increasing rolling reduction from 20 to $80 \%$, the increment in the UTS is nearly the same, whereas the elongation to failure decreased from $36 \%$ to $4 \%$, respectively.

\section{Acknowledgements}

The financial support received from the Ministry of Education and Science, Russia, under grant №14.A18.21.1213 is gratefully acknowledged. One of the authors (DM) expresses his gratitude to the Deutsche Forschungsgemeinschaft (DFG) for financial support within the Collaborative Research Centre (SFB) 761 "Stahl ab-initio. Quantenmechanisch geführtes Design neuer Eisenbasiswerkstoffe". The authors are grateful to the personnel of the Joint Research Centre, Belgorod State University, for their assistance with instrumental analysis.

\section{References}

[1] O. Bouaziz, S. Allain, C.P. Scott, P. Cugya, D. Barbier, Current Opinion in Solid State and Materials Science 15 (2011) 141-168.

[2] B. C. De Cooman, Kwang-geun Chin and Jinkyung Kim, in: Marcello Chiaberge (ed.), New Trends and Developments in Automotive System Engineering, InTech, 2011, pp. 101-128.

[3] O. Grassel, L. Kruger, G. Frommeyer, L.W. Meyer, Int. J. Plast. 16 (2000) 1391-1409.

[4] I. Gutierrez-Urrutia, D. Raabe, Acta Mat. 59 (2011) 6449-6462.

[5] A. Dumay, J.-P. Chateau, S. Allain, S. Migot, O. Bouaziz, , Mat. Sci. Eng. A 483-484 (2008) $184-187$.

[6] O. Bouaziz, N. Guelton, Mater. Sci. Eng. A 319-321 (2001) 484-487.

[7] Y. P. Lü, D. A. Molodov, G. Gottstein, ISIJ Int. 51 (2011) 812-817.

[8] C. Haase, S. G. Chowdhury, L. A. Barrales-Mora, D. A. Molodov, G. Gottstein, Metall. Mater. Trans. A 44A (2012) 911-922.

[9] R.E. Smallman, K.H. Westmacott, Philos. Mag. 2 (1957) 669-683.

[10] I. Shakhova, V. Dudko, A. Belyakov, K. Tsuzaki, R. Kaibyshev, Mater. Sci. Eng. A 545 (2012) 176-186.

[11] R. Z.Valiev, T. G. Langdon, Adv. Eng. Mater. 12 (2010) 677-691. 